\title{
Multifocal atrial tachycardia in two neonates
}

\author{
ZIA Q. FAROOKI AND EDWARD W. GREEN \\ From the Department of Pediatrics, Wayne State University School of Medicine and Children's \\ Hospital of Michigan, Detroit, Michigan, U.S.A.
}

Two neonates with rapid and irregular pulse rate had an uncommon form of atrial tachycardia. The irregular heart rate was first detected during fetal monitoring. Postnatal electrocardiograms were compatible with the diagnosis of multifocal atrial tachycardia or chaotic atrial rhythm. Both patients were treated with digoxin and the rhythm gradually reverted to sinus. This may represent the first description of multifocal atrial tachycardia in the newborn.

Multifocal atrial tachycardia is an uncommon form of atrial arrhythmia which results in irregularly irregular pulse. This unusual form of supraventricular tachycardia is usually seen in elderly patients with lung disease. To the best of our knowledge, multifocal atrial tachycardia has not been reported in neonates. This report describes two asymptomatic neonates who presented with rapid and irregularly irregular heart rate and who were found to have multifocal atrial tachycardia.

\section{Case reports}

CASE 1

A 4-day-old full-term male infant was transferred to Children's Hospital of Michigan because of rapid and irregular heart rate. The pregnancy was uneventful. The mother had been treated with three grains of dessicated thyroid daily during pregnancy and for 19 years previously. Labour was induced because of irregular fetal heart tones, with an average rate of 100 per minute. Apart from the irregular heart rhythm, the physical examination was normal. There was no evidence of congestive heart failure. The chest $x$-ray film showed normal heart size, normal vascularity, and left aortic arch. The electrocardiogram disclosed right axis deviation, an rsR' pattern in V3R and V1 and chaotic atrioventricular rhythm. Fig. 1 (A and B) shows selected strips of lead $\mathrm{V} 1$. The average atrial rate was 250 per minute, and the ventricular rate ranged between 150 and 250 per minute. The PP, $R R$, and $P R$ intervals were variable. There were 5 different types of $P$ waves present ( $a, b, c, d$, and $e$ ), with a distinct isoelectric baseline between them. The arrow in Fig. $1 \mathrm{~B}$ shows a nonconducted atrial Received for publication 14 December 1976 premature depolarisation and aberrantly depolarised ventricular complexes.

The patient was given oral digoxin, which produced gradual slowing of the ventricular rate and decreasing atrial ectopy. The electrocardiogram on the third day of treatment (Fig. $1 \mathrm{C}$ ) showed three types of $P$ waves $(a, b, d)$, the atrial rate ranged between 150 and 250 per minute, the ventricular rate ranged between 90 and 180 per minute, and there was an occasional ventricular complex with aberrant conduction. The laboratory data including microprotein-bound iodine were normal. One week after digitalis the electrocardiogram showed sinus rhythm with right bundle-branch block pattern. At 1 year of age digitalis was stopped, and no arrhythmias occurred during the subsequent 2 years of follow-up.

\section{CASE 2}

A 24-hour-old full-term male infant was referred to Children's Hospital of Michigan because of an irregular and rapid heart rate. The pregnancy was uncomplicated. Labour had been induced because of irregular fetal heart sounds. The physical examination was normal except for an irregularly irregular heart rate ranging between 120 and 150 per minute. There was no congestive heart failure. The chest $x$-ray film disclosed normal heart size, normal vascularity, and left aortic arch. The electrocardiogram showed right axis deviation with no evidence of chamber enlargement. Fig. 2 shows the selected strips of lead II; Fig. 2 A and B were obtained on admission, when the atrial rate was rapid and irregular (range: 115 to 500 per minute). The ventricular rate was irregular and rapid (range: 115 to 200 per minute). The PP, RR, and PR intervals were variable with 3 different types of $P$ waves 


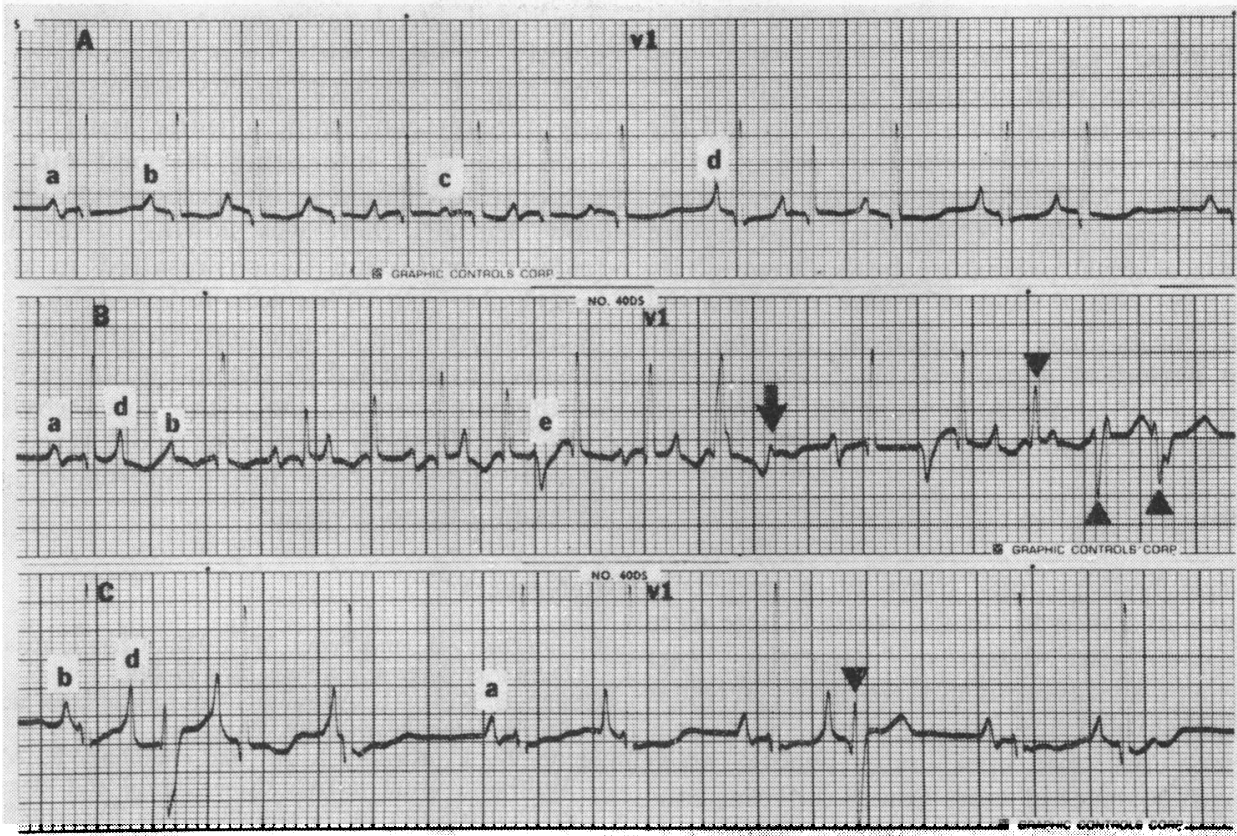

Fig. 1 Electrocardiogram recorded at $50 \mathrm{~mm} / \mathrm{s} .(A)$ and $(B)$ are the strips of lead $V 1$ recorded on admission. The $P P, P R$, and $R R$ intervals are variable, and 5 types of $P$ waves $(a, b, c, d, e)$ are present. The arrow points toward nonconducted atrial premature depolarisation. The triangles point toward aberrantly conducted ventricular complexes. (C) A strip of lead V1 recorded at double sensitivity $(1 \mathrm{mV}=20 \mathrm{~mm})$ after digitalis. There are 3 types of $P$ waves $(a, b, d)$ present. The ventricular rate has decreased.

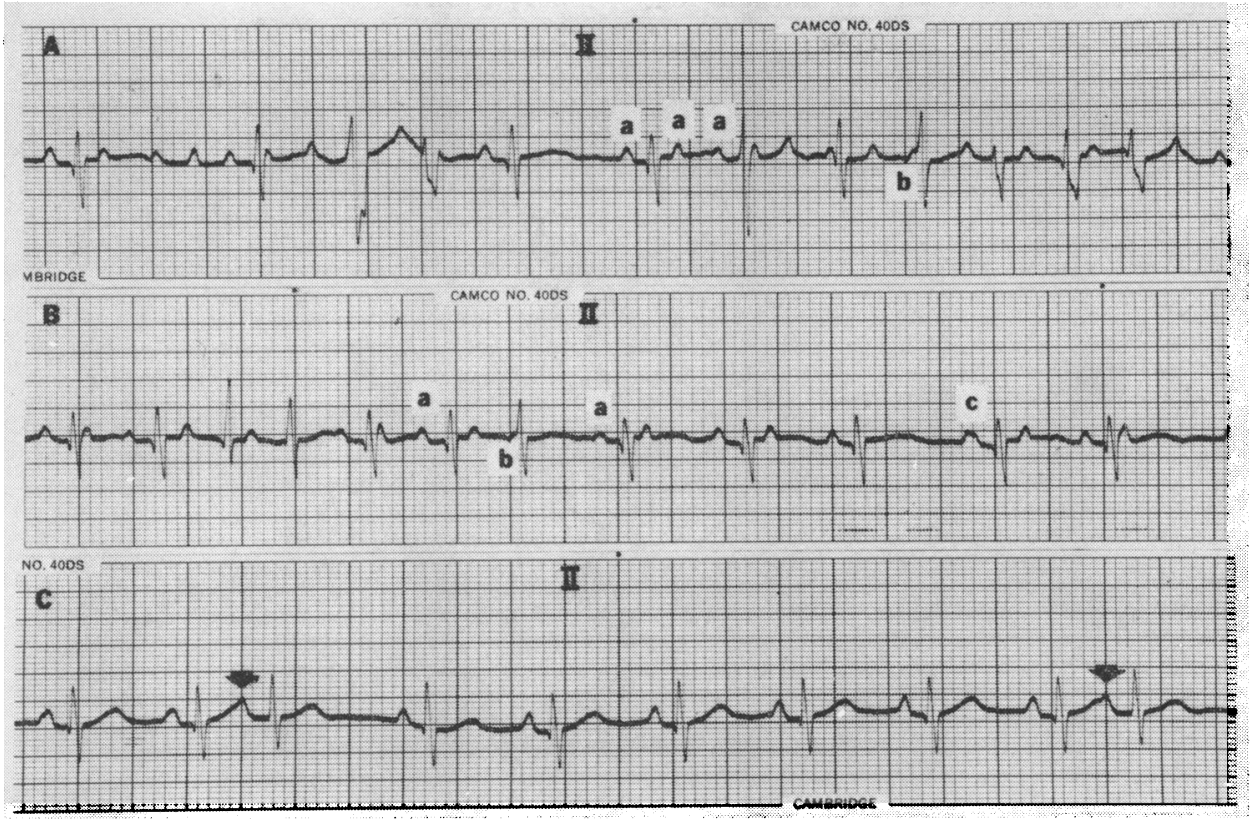

Fig. 2 Electrocardiogram recorded at $50 \mathrm{~mm}$ per second. (A) and (B) Strips of lead V1 recorded on admission. $P P, P R$, and $R R$ intervals are variable, and 3 types of $P$ wave $(a, b, c)$ are present. (C) Strip of lead V1 recorded after digitalisation. There are unifocal atrial premature beats (arrows) present. 
(a, o, c). There was a distinct isoelectric interval present between the $d$ waves. There were ventricular complexes with aberrant conduction.

The baby was treated with oral digoxin and the ectopic atrial activity was significantly depressed by the fourth day. The electrocardiogram after 1 week of digoxin therapy (Fig. 2 C) showed sinus rhythm with uniforcal atrial premature beats (arrows). By the end of the second week of treatment, the atrial premature beats had disappeared. During the follow-up period of approximately 5 months the rhythm remained sinus. At present the baby is on digoxin and is asymptomatic.

\section{Comments}

Both babies had irregular heart rates prenatally and this was mistaken for fetal distress. There were no associated heart defect or congestive heart failure. The pulse was rapid and irregularly irregular and the electrocardiogram in both cases was characterised by: fast atrial rate, chaotic atrial rhythm with $P$ waves of more than 3 different configurations, the presence of an isoelectric baseline between the $P$ waves, and a variable degree of atrioventricular block. These electrocardiographic features fulfil the criteria for the diagnosis of multifocal atrial tachycardia or chaotic atrial rhythm. The published reports (Shine et al., 1968; Chung, 1971; Berlinerblau and Feder, 1972) indicate that multifocal atrial tachycardia is usually seen in elderly patients with bronchopulmonary disease and digitalis intoxication. We were unable to find any reports concerning chaotic atrial rhythm in neonates. It is interesting to point out that the response to digoxin in our cases was complete but slow. Initially there was slowing of the atrioventricular conduction which resulted in decreased ventricular rate. This was followed by a gradual decrease in atrial ectopic activity and sinus rhythm was well established by the end of the second week of digoxin therapy. Once the sinus rhythm had been established, recurrence of ectopic atrial activity did not occur during the follow-up period extending up to 3 years.

\section{References}

Berlinerblau, R., and Feder, W. (1972). Chaotic atrial rhythm. fournal of Electrocardiology, 5, 135-144.

Chung, E. K. (1971). Appraisal of multifocal atrial tachycardia. British Heart fournal, 33, 500-504.

Shine, K. I., Kastor, J. A., and Yurchak, P. M. (1968). Multifocal atrial tachycardia-Clinical and electrocardiographic features in 32 patients. New England fournal of Medicine, 279, 344-349.

Requests for reprints to Dr. Zia Q. Farooki, Cardiology Section, Children's Hospital of Michigan, 3901 Beaubien Boulevard, Detroit, Michigan 48201, U.S.A. 